OPEN ACCESS

Edited by:

Ziad Daoud,

Central Michigan University,

United States

Reviewed by:

Balaji Veeraraghavan,

Christian Medical College \& Hospital, India

Prakash Sah,

University of Oklahoma Health Sciences Center, United States

*Correspondence:

Spyros Pournaras spournaras@med.uoa.gr

Specialty section: This article was submitted to

Clinical Microbiology,

a section of the journal

Frontiers in Cellular and

Infection Microbiology

Received: 13 November 2021

Accepted: 28 December 2021

Published: 20 January 2022

Citation:

Petropoulou D, Siopi M, Vourli S and Pournaras S (2022)

Activity of SulbactamDurlobactam and Comparators Against a National Collection of Carbapenem-Resistant Acinetobacter baumannii Isolates From Greece. Front. Cell. Infect. Microbiol. 11:814530. doi: 10.3389/fcimb.2021.814530

\section{Activity of Sulbactam-Durlobactam and Comparators Against a National Collection of Carbapenem-Resistant Acinetobacter baumannii Isolates From Greece}

\author{
Dimitra Petropoulou, Maria Siopi, Sophia Vourli and Spyros Pournaras* \\ Laboratory of Clinical Microbiology, Attikon University Hospital, School of Medicine, National and Kapodistrian University of \\ Athens, Athens, Greece
}

Background: Acinetobacter baumannii is a leading cause of healthcare-associated infections worldwide, due to both its persistence in the hospital setting and ability to acquire high levels of antibiotic resistance. Carbapenem-resistant $A$. baumannii isolates (CRAB) limit the activity of current antimicrobial regimens and new alternatives or adjuncts to traditional antibiotics are urgently needed. Durlobactam is a novel broad-spectrum inhibitor of serine-type $\beta$-lactamases that restores sulbactam (SUL) activity against $A$. baumannii. The sulbactam-durlobactam (SD) combination has recently completed Phase 3 testing in the global ATTACK trial.

Objectives: The aim of this study is to evaluate the in vitro activity of SD versus comparators against a representative nationwide collection of CRAB isolates.

Methods: One hundred ninety CRAB isolates were collected from clinical samples of patients hospitalized in 11 hospitals throughout Greece during 2015. In vitro activities of SD and comparators (SUL alone, amikacin, minocycline, imipenem, meropenem, colistin, SD and imipenem combined with SD) were determined by broth microdilution.

Results: Durlobactam restored sulbactam activity against the majority of the strains tested, with $\mathrm{SD}$ exhibiting the lowest $\mathrm{MIC}_{90}(8 \mu \mathrm{g} / \mathrm{ml})$ relative to the other single comparators tested; $87.9 \%$ of the isolates had SD MICs $\leq 4 / 4 \mu \mathrm{g} / \mathrm{ml}$. The most active comparator was colistin $\left(\mathrm{MIC}_{90}=16 \mu \mathrm{g} / \mathrm{ml}\right)$. The addition of imipenem further lowered the $\mathrm{MIC}_{90}$ of SD by one two-fold dilution.

Conclusions: This study demonstrated the potential utility of SD for the treatment of infections caused by $A$. baumannii. If its clinical efficacy is confirmed, SD may be an important therapeutic option for CRAB infections.

Keywords: hospital infections, diazabicyclooctane, durlobactam, carbapenemases, beta-lactamase inhibitor, serine-type beta-lactamases, CRAB infections, sulbactam-durlobactam-imipenem 


\section{INTRODUCTION}

Infections caused by multidrug-resistant (MDR) A. baumannii pose a serious threat to global health. These infections include ventilator-associated pneumonia, bacteremia, complicated urinary tract infections and skin and soft tissue infections, in both healthy and immuno-compromised individuals (Lee et al., 2017). The mortality rates of these infections reach $33 \%$, and carbapenem resistance was associated with a greater risk of death (pooled odds ratio 2.22, 95\% CI 1.66-2.98) [European Centre for Disease Prevention and Control (ECDC), 2013; Lemos et al., 2014]. A recent analysis of the global prevalence of antibiotic resistance in $A$. baumannii infections found a prevalence of resistance to imipenem of 73.9-77.8\%, which represented a dramatic increase since 2005 (Xie et al., 2018).

The epidemiological status of carbapenem-resistant $A$. baumannii (CRAB) in Greece is defined as endemic situation, with most hospitals repeatedly facing cases admitted from endemic sources [European Centre for Disease Prevention and Control (ECDC), 2013]. The resistance to carbapenems in $A$. baumannii precludes the use of $\beta$-lactam therapies; the main mechanism responsible is the production of oxacillinases (class D $\beta$-lactamases), with the most frequent ones being OXA-23, OXA24/40 and OXA-58 (D'Arezzo et al., 2010; Gogou et al., 2011; Schleicher et al., 2013). Among the limited $\beta$-lactams that retain a degree of activity against A. baumannii is sulbactam (SUL), a semisynthetic penicillanic acid, which is a first-generation $\beta$-lactamase inhibitor, with limited activity against class A serine $\beta$-lactamases (Seifert et al., 2020; Shapiro et al., 2021). SUL inhibits A. baumannii enzymes that are involved in bacterial peptidoglycan synthesis, such as PBP1a, PBP1b and PBP3 (Higgins et al., 2014). However, due to its susceptibility to degradation by a variety of acquired or upregulated $\beta$-lactamases, SUL has limited clinical utility against infections caused by CRAB (Penwell et al., 2015).

A promising new therapeutic option for $\mathrm{CRAB}$ is the combination of sulbactam with durlobactam (SD), a new member of the diazabicyclooctane class of $\beta$-lactamase inhibitors, with broad spectrum activity against Ambler class $A, C$ and $D$ serine $\beta$ lactamases, resulting in the restoration of the susceptibility of CRAB isolates to $\beta$-lactams (Durand-Réville et al., 2017). Durlobactam (DUR) restores SUL susceptibility of $A$. baumannii strains overexpressing individual $\beta$-lactamases (Seifert et al., 2020; Shapiro et al., 2021) as well as diverse clinical isolates, in international, contemporary surveillance studies (McLeod et al., 2020; Yang et al. 2020; Nodari et al. 2021). SD is currently in late-stage development for the treatment of infections caused by Acinetobacter spp.

The aim of this study was to examine the in vitro potency of the SD combination on a collection of previously characterized, non-duplicate isolates of CRAB from Greece harboring acquired $\beta$-lactamases.

\section{MATERIALS AND METHODS}

\section{Bacterial Strains}

The study included 190 non-repetitive CRAB isolates recovered during 2015 from 11 geographically distinct tertiary hospitals, located throughout Greece and selected during a previous nationwide study randomly from a collection of 2,500 A. baumannii isolates. The clinical samples included blood, bronchial aspirates, urine, superficial or deep tissue wounds, peritoneal and pleural effusions, cerebrospinal fluids and intraabdominal secretions. All isolates were previously characterized and confirmed to be A. baumannii by PCR/sequencing for the

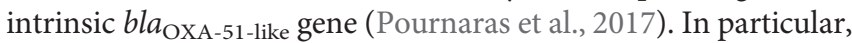
clonality was tested by a scheme based on two multiplex PCRs, that selectively amplified alleles of the $\operatorname{omp} A, c s u E$ and

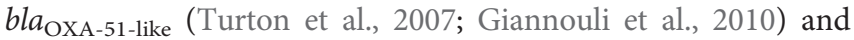
single-locus bla ${ }_{\text {OXA-51-like }}$ sequence-based typing (SBT) (Pournaras et al., 2014). The SBT assigned 153 isolates (80.5\%) to IC2, 36 isolates to IC1 (18.9\%) and 1 isolate to G6 (0.5\%). Of the 153 IC2 isolates, all had bla $a_{\text {OXA-23-like }}$ and three had both

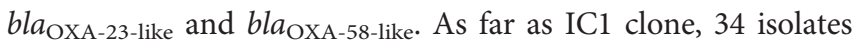
(94.5\%) had bla $a_{\text {OXA-23-like }}$ and two (5.5\%) had bla $a_{\text {OXA-58-like. }}$ As for the $\beta$-lactamases carried by the study isolates,

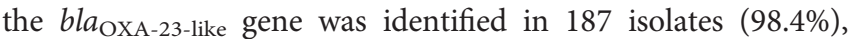
$b l a_{\text {OXA-23-like }}$ together with $b l a_{\text {OXA-58-like }}$ in three $(1.6 \%), b l a_{\text {OXA }}$ 58 -like in two $(1.0 \%)$ and $b l a_{\text {OXA-40-like }}$ in one isolate $(0.5 \%)$.

\section{Antimicrobial Susceptibility}

Antimicrobial susceptibility was determined using broth microdilution in freshly prepared cation-adjusted MuellerHinton broth (CAMHB) following CLSI recommendations (CLSI, 2018; CLSI, 2019). Pre-manufactured, frozen 96-well plates containing $50 \mu \mathrm{L}$ of $2 \mathrm{x}$ antimicrobial drug concentrations were supplied by Entasis Therapeutics. The 190 CRAB isolates were tested against SUL, amikacin (AMK), minocycline (MIN), imipenem (IMP), meropenem (MER), colistin (COL), SD and IMP combined with SD (SID). The concentration ranges tested in 2-fold dilutions were for SUL, 0.06 to $64 \mu \mathrm{g} / \mathrm{ml}$; SD [durlobactam (DUR) fixed at $4 \mu \mathrm{g} / \mathrm{ml}$ ], $0.06 / 4$ to $64 / 4 \mu \mathrm{g} / \mathrm{ml}$; SID (1/1/2 ratio), 0.06/0.06/0.12 to 64/64/128; AMK, 0.12 to $128 \mu \mathrm{g} / \mathrm{ml}$; COL, 0.06 to $64 \mu \mathrm{g} / \mathrm{ml}$; IMP, 0.06 to $64 \mu \mathrm{g} / \mathrm{ml}$; MER, 0.06 to $64 \mu \mathrm{g} / \mathrm{ml}$; and MIN, 0.03 to $32 \mu \mathrm{g} / \mathrm{ml}$. The combination of SUL, IMP and DUR was tested in a fixed 1:1:2 ratio titrated in 2-fold dilutions. MICs were interpreted according to CLSI guidelines and susceptibilities were determined using CLSI breakpoints, where applicable. Each experiment included testing of CLSI-approved quality control organisms NCTC 13304 (A. baumannii), ATCC 25922 (Escherichia coli) and ATCC 27853 (Pseudomonas aeruginosa). The minimal inhibitory concentration (MIC) of each antibiotic was determined by visual inspection for each strain after incubation for 20 hours at $35^{\circ} \mathrm{C}$.

\section{Next Generation Sequencing of Isolates With Elevated SD MICs}

Three isolates with SD MICs $>8 / 4 \mu \mathrm{g} / \mathrm{ml}$ were analyzed by next Generation Sequencing. Genomic DNA was purified from the isolates using a Sigma-Aldrich GenElute bacterial genomic DNA kit. Genomic libraries were assembled using a Nextera XT library preparation kit and sequenced using an Illumina MiSeq system with 300-bp paired-end reads and a coverage of $\geq 50 \mathrm{X}$. Assembly 
and analysis of the whole genome sequencing was performed using CLC Genomics Workbench v21.0.3 (Qiagen, Germantown, MD). Paired Fastq files were processed and analyzed as follows: raw reads were trimmed of any remaining barcode sequences as well as trimmed for quality. Reads were then de novo assembled using fraction length $=0.8$ and similarity fraction $=0.9$ using default mismatch/insertion/deletion costs. Consensus sequences were extracted and contigs greater than $500 \mathrm{bp}$ were assembled. B-lactamase genes for each strain were identified by BLAST against a local $\beta$-lactamase database. Additionally, mutations in efflux, permeation, and PBP proteins were identified by BLAST analysis against the $A$. baumannii ATCC 17978 reference strain. The genomic sequences of the three strains tested were deposited at http:// www.ncbi.nlm.nih.gov/bioproject/781741.

\section{RESULTS}

The SD $\mathrm{MIC}_{50 / 90}$ values were $4 / 4$ and $8 / 4 \mu \mathrm{g} / \mathrm{ml}$, respectively. The SID MIC $50 / 90$ values were $2 / 2 / 4$ and $4 / 4 / 8 \mu \mathrm{g} / \mathrm{ml}$, respectively (Table 1). The $\mathrm{MIC}_{50 / 90}$ values of currently-used comparator antimicrobials were: SUL (64/>64), COL (2/16), MIN (16/32), IMP $(>64 />64)$, MER $(>64 />64)$, and AMK $(>128 />128) \mu \mathrm{g} / \mathrm{ml}$ (Table 2).

All isolates had IMP and MER MICs $\geq 32 \mu \mathrm{g} / \mathrm{ml}$. Resistance rates to comparators were as follows: IMP, 100\%; MER, 100\%; AMK, 97.4\%; and MIN, 57.3\%. Of concern, 61 of the 190 (32.1\%) isolates were resistant to COL and among isolates from blood cultures, the resistance rate reached $36 \%$. Among the COLresistant isolates, 54 (88.5\%) had low SD MICs of $\leq 4 \mu \mathrm{g} / \mathrm{ml}$ and all (100\%) had SID MICs of $\leq 4 / 4 / 8 \mu \mathrm{g} / \mathrm{ml}$.

Of the isolates, $87.9 \%$ had SD MICs of $\leq 4 / 4 \mu \mathrm{g} / \mathrm{ml}$ and only three isolates had SD MIC $>8 \mu \mathrm{g} / \mathrm{ml}$. These three isolates were submitted to NGS sequencing. The genomic characteristics of these isolates are shown in Table 3. In brief, they belonged to three different MLST types (ST-1834, SD MIC $16 \mu \mathrm{g} / \mathrm{ml}$; ST1294 SD MIC >64 $\mu \mathrm{g} / \mathrm{ml}$; and ST-425, SD MIC $16 \mu \mathrm{g} / \mathrm{ml})$. They all carried $b l a_{\text {OXA-23 }}$ and $b l a_{\text {OXA-66 }}$ and all encoded the same A515V variant of the PBP3 gene that likely confers resistance to SUL, considering its proximity to the SUL binding site (PappWallace et al., 2012). The SID MIC for both ST-1834 and ST-425 isolates was $4 \mu \mathrm{g} / \mathrm{ml}$ (i.e. addition of IMP helped reduce the SD MIC), while the ST-1294 isolate had SD MIC of $>64 \mu \mathrm{g} / \mathrm{ml}$ and SID MIC $64 / 64 / 128 \mu \mathrm{g} / \mathrm{ml}$. The latter isolate also harbored the NDM metallo- $\beta$-lactamase gene, which DUR does not inhibit.

\section{DISCUSSION}

The most prevalent mechanism of carbapenem resistance among A. baumannii is associated with carbapenem-hydrolysing enzymes that belong to Ambler class D and B $\beta$-lactamases (Jeon et al., 2015; Lee et al., 2017; Wong et al., 2017; Lötsch et al., 2020). The rapid rise of carbapenem resistance among $A$. baumannii isolates limits the available therapeutic options and poses a serious need for new antimicrobial agents. In particular, according to 2019 data from the European Antimicrobial Resistance Surveillance Network (EARS-Net), nearly a third of invasive Acinetobacter spp. isolates in the EU/EEA are already resistant to carbapenems [European Centre for Disease Prevention and Control (ECDC), 2019; Lötsch et al., 2020]. Carbapenem resistance rates are higher than $50 \%$ in southern and eastern European countries [European Centre for Disease Prevention and Control (ECDC), 2019], while in Greece, according to data from the Hellenic CDC, IMP resistance rates are currently exceeding 90\% (WHONET). Of note, in our representative countrywide collection, $\mathrm{CRAB}$ isolates exhibited particularly high levels of resistance to last-line antimicrobials in addition to carbapenems, including AMK, SUL, MIN and COL.

Among the few therapeutic options that show efficacy against $\mathrm{CRAB}$ isolates is COL, used both as monotherapy or in combination with other antimicrobials. Still, while COL is a key drug, there are concerns, not only about its toxicity profiles, but also its rising resistance rates (Viehman et al., 2014). A metaanalysis on the prevalence of A. baumanni antimicrobial resistance worldwide from 2000 to 2017, showed that the overall global resistance rate reaches $11.2 \%$ (Pormohammad et al., 2020). Herein, COL showed a much higher resistance rate of $32.1 \%$ and among isolates, collected from blood cultures, the rate reached $36 \%$.

The carbapenem resistance problem of A. baumannii can be overcome by the use of expanded-spectrum serine $\beta$-lactamase inhibitors, which may inhibit class $A, C$ or $D \beta$-lactamases, resulting in restoration of $\beta$-lactam activity. SD is a promising combination and its spectrum of activity can address MDR $A$. baumannii. In our study, the addition of DUR at a fixed concentration of $4 \mu \mathrm{g} / \mathrm{ml}$ lowered the $\mathrm{MIC}_{50}$ and $\mathrm{MIC}_{90}$ of SUL from 64 and $>64$ to 4 and $8 \mu \mathrm{g} / \mathrm{ml}$, respectively, except for the isolate that encoded metallo- $\beta$-lactamase. The $\mathrm{MIC}_{90}$ of $\mathrm{SD}$ was considerably lower than the $\mathrm{MIC}_{90}$ of carbapenems, MIN and AMK and also lower from that of COL $[16 \mu \mathrm{g} / \mathrm{ml}] .88 .5 \%$ of non-susceptible to COL isolates had SD MICs $\leq 4 \mu \mathrm{g} / \mathrm{ml}$.

A. baumannii isolates belonging to IC1 showed generally more sensitive profiles compared to IC2, concerning SUL, COL

TABLE 1 | MIC distribution of the 190 CRAB isolates for SUL, SD and SID and their MIC ${ }_{50}$, MIC $\mathrm{C}_{90}$ values.

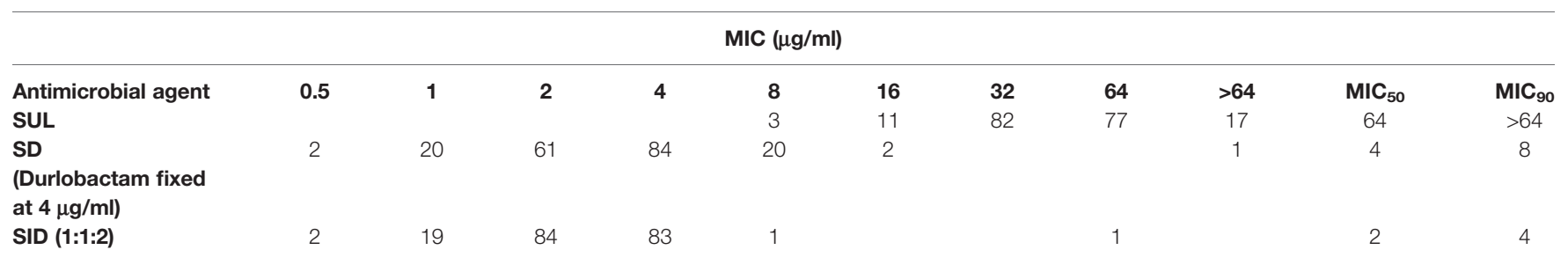


TABLE 2 | Resistance rates of the 190 CRAB isolates and their international clonal lineages IC1 and IC2 for SUL, COL, IMP, MER, AMK, MIN, SD and SID and their $\mathrm{MIC}_{50}, \mathrm{MIC}_{90}$ values.

\begin{tabular}{|c|c|c|c|c|c|c|c|c|c|c|c|c|c|c|c|}
\hline \multirow[t]{2}{*}{ Antimicrobial agent } & \multicolumn{5}{|c|}{ OVERALL $(n=190)$} & \multicolumn{5}{|c|}{ IC1 $(n=36)$} & \multicolumn{5}{|c|}{ IC2 $(n=153)$} \\
\hline & $\mathrm{MIC}_{50}$ & $\mathrm{MIC}_{90}$ & S \% & $1 \%$ & $\mathbf{R} \%$ & $\mathrm{MIC}_{50}$ & $\mathrm{MIC}_{90}$ & $\mathrm{~S} \%$ & $1 \%$ & $\mathbf{R} \%$ & $\mathrm{MIC}_{50}$ & $\mathrm{MIC}_{90}$ & $\mathrm{~S} \%$ & $1 \%$ & $\mathbf{R} \%$ \\
\hline SUL & 64 & $>64$ & - & & - & 32 & 64 & - & & - & 64 & $>64$ & - & & - \\
\hline COL & 2 & 16 & 67.9 & 0 & 32.1 & 1 & 16 & 75 & 0 & 25 & 2 & 16 & 66 & 0 & 34 \\
\hline IMP & $>64$ & $>64$ & 0 & 0 & 100 & 64 & $>64$ & 0 & 0 & 100 & $>64$ & $>64$ & 0 & 0 & 100 \\
\hline MER & $>64$ & $>64$ & 0 & 0 & 100 & 64 & $>64$ & 0 & 0 & 100 & $>64$ & $>64$ & 0 & 0 & 100 \\
\hline AMK & $>128$ & $>128$ & 2.1 & 0.5 & 97.4 & $>128$ & $>128$ & 2,8 & 0 & 97,2 & $>128$ & $>128$ & 2 & 0,7 & 97,3 \\
\hline MIN & 16 & 32 & 25.3 & 17.4 & 57.3 & 2 & 4 & 97,2 & 0 & 2,8 & 16 & 32 & 7,8 & 21 & 71,2 \\
\hline SD (Durlobactam fixed at $4 \mu \mathrm{g} / \mathrm{ml}$ ) & 4 & 8 & - & & - & 4 & 4 & - & & - & 4 & 4 & - & & - \\
\hline SID $(1: 1: 2)$ & 2 & 4 & - & & - & 2 & 4 & & & & 2 & 4 & & & \\
\hline
\end{tabular}

TABLE 3 | Accession numbers and resistance mechanisms detected by next generation sequencing of the three isolates with SD MICs > 8 Mg/ml.

\begin{tabular}{|c|c|c|c|c|c|c|c|c|c|}
\hline \multirow{2}{*}{$\begin{array}{l}\text { Genome } \\
\text { accession }\end{array}$} & \multirow{2}{*}{$\begin{array}{l}\text { SD MICs } \\
(\mu \mathrm{g} / \mathrm{ml})\end{array}$} & \multirow{2}{*}{$\begin{array}{l}\text { SID MICs } \\
(\mu \mathrm{g} / \mathrm{ml})\end{array}$} & \multirow{2}{*}{$\begin{array}{c}\text { MLST } \\
\text { Classification }\end{array}$} & \multicolumn{4}{|c|}{ Encoded BLAs } & \multicolumn{2}{|c|}{ Other mutations } \\
\hline & & & & $\begin{array}{c}\text { Class } \\
\text { A }\end{array}$ & $\begin{array}{c}\text { Class } \\
\text { B }\end{array}$ & Class C & Class D & PBPs & $\begin{array}{c}\text { efflux } \\
\text { components }\end{array}$ \\
\hline JAJKGX000000000 & 16 & $4 / 4 / 8$ & $\begin{array}{c}\text { ST-1834, } 436 / \mathrm{PST}- \\
2\end{array}$ & TEM-1 & - & ADC-73 & $\begin{array}{c}\text { OXA-23; OXA- } \\
66\end{array}$ & $\begin{array}{c}\text { PBP3 } \\
\text { [A515V] }\end{array}$ & - \\
\hline JAJKGWW000000000 & $>64$ & $64 / 64 / 128$ & ST-1294/PST-570 & TEM-1 & NDM-1 & ADC-73 & $\begin{array}{c}\text { OXA-23; OXA- } \\
66\end{array}$ & $\begin{array}{l}\text { PBP3 } \\
\text { [A515V] }\end{array}$ & - \\
\hline JAJKGV000000000 & 16 & $4 / 4 / 8$ & ST-425/PST-2 & - & - & $\begin{array}{c}\text { ADC- } \\
188\end{array}$ & $\begin{array}{c}\text { OXA-23; OXA- } \\
66\end{array}$ & $\begin{array}{l}\text { PBP3 } \\
{[\mathrm{A} 515 \mathrm{~V}]}\end{array}$ & AdeR [G25S] \\
\hline
\end{tabular}

and carbapenems. For MIN, the resistance rates between IC1 and IC2 isolates were $2.8 \%$ and $71.2 \%$, respectively. There was no difference on the MICs of SD and SID among the isolates of the two clonal lineages. For both IC2 and IC2 isolates, $\mathrm{MIC}_{50 / 90}$ of SD and SID were at $4 / 4$ and $2 / 4$, respectively.

Compared to other studies on the activity of SD, our collection of isolates presented higher resistance rates and MICs for the comparators, as well as for SD. In particular, both the $\mathrm{SD} \mathrm{MIC}_{50}$ and $\mathrm{MIC}_{90}$ of the strains in the present study exceeded by one to three-fold the respective values of those reported in international studies (McLeod et al., 2020; Seifert et al., 2020; Yang et al., 2020; Nodari et al., 2021), indicating the presence of less susceptible strains in Greek hospitals. COL MICs were also considerably lower in three of those studies $\left(\mathrm{MIC}_{90}\right.$ $1 \mu \mathrm{g} / \mathrm{ml}$ in references Higgins et al., 2004; Pournaras et al., 2017; Seifert 2020), with only one study from China reporting COL $\mathrm{MIC}_{90} 128 \mu \mathrm{g} / \mathrm{ml}$ (Durand-Réville et al., 2017). Interestingly, the addition of IMP to SD lowered its MIC90 by one twofold dilution.

Our study clearly showed that SD had excellent in vitro activity against CRAB isolates that were highly resistant to IMP, MER, AMK, MIN and COL. In addition, SD showed favorable clinical efficacy and safety in a recently completed, global phase 3 study (Entasis Therapeutics, 2019), https://investors.entasistx.com/ news-releases/news-release-details/entasis-therapeuticsannounces-positive-topline-results). If approved this combination may provide an important therapeutic option for infections due to MDR A. baumannii, including CRAB.
Part of this work was presented at the 31st European Congress of Clinical Microbiology and Infectious Diseases (ECCMID), Online, 9-12 July 2021.

\section{DATA AVAILABILTY STATEMENT}

The raw data supporting the conclusions of this article will be made available by the authors, without undue reservation.

\section{AUTHOR CONTRIBUTIONS}

All authors listed have made a substantial, direct and intellectual contribution to the work, and approved it for publication.

\section{FUNDING}

The research being submitted received a grant from ENTASIS Therapeutics. This grant did not include open access publication fees, which will be paid by the research account of our Laboratory in our institution (National and Kapodistrian University of Athens Special Account for Research Grants).

\section{ACKNOWLEDGMENTS}

We thank Samir Moussa of Entasis Therapeutics for next generation sequencing and analysis. 


\section{REFERENCES}

CLSI. (2018). Methods for Dilution Antimicrobial Susceptibility Tests for Bacteria That Grow Aerobically, Eleventh Edition. CLSI standard M07. Wayne, PA: Clinical and Laboratory Standards Institute.

CLSI. (2019). Performance Standards for Antimicrobial Susceptibility Testing, Twenty-Ninth Edition. CLSI supplement M100. Wayne, PA: Clinical and Laboratory Standards Institute.

D'Arezzo, S., Principe, L., Capone, A., Petrosillo, N., Petrucca, A., and Visca, P. (2010). Changing Carbapenemase Gene Pattern in an Epidemic Multidrug-Resistant Acinetobacter Baumannii Lineage Causing Multiple Outbreaks in Central Italy. J. Antimicrob. Chemother. 66, 54-61. doi: $10.1093 / \mathrm{jac} / \mathrm{dkq} 407$

Durand-Réville, T., Guler, S., Comita-Prevoir, J., Chen, B., Bifulco, N., Huynh, H., et al. (2017). ETX2514 Is a Broad-Spectrum $\beta$-Lactamase Inhibitor for the Treatment of Drug-Resistant Gram-Negative Bacteria Including Acinetobacter Baumannii. Nat. Microbiol. 2, 17104. doi: 10.1038/nmicrobiol.2017.104

Entasis Therapeutics. (2019). Study to Evaluate the Efficacy and Safety of Intravenous Sulbactam-ETX2514 in the Treatment of Patients With Infections Caused by Acinetobacter Baumannii-Calcoaceticus Complex (ATTACK). Available at: https://clinicaltrials.gov/ct2/show/NCT03894046? term $=$ sulbactam\&draw $=4 \&$ rank $=21$.

European Centre for Disease Prevention and Control (ECDC). (2013). Carbapenemase-Producing Bacteria in Europe - Interim Results From the European Survey on Carbapenemase-Producing Enterobacteriaceae (EuSCAPE) Project 2013 (Stockholm: ECDC). Available at: https://ecdc. europa.eu/sites/portal/files/media/en/publications/Publications/antimicrobialresistance-carbapenemase-producing-bacteria-europe.

European Centre for Disease Prevention and Control (ECDC). (2019). Surveillance of Antimicrobial Resistance in Europe 2018. Annual Report of the European Antimicrobial Resistance Surveillance Network (EARS-Net) (Stockholm: ECDC). Available at: https://www.ecdc.europa.eu/sites/default/ files/documents/surveillance-antimicrobial-resistance-Europe-2018.

Giannouli, M., Cuccurullo, S., Crivaro, V., Di Popolo, A., Bernardo, M., Tomasone, F., et al. (2010). Molecular Epidemiology of Multidrug-Resistant Acinetobacter Baumannii in a Tertiary Care Hospital in Naples, Italy, Shows the Emergence of a Novel Epidemic Clone. J. Clin. Microbiol. 48, 1223-1230. doi: 10.1128/JCM.02263-09

Gogou, V., Pournaras, S., Giannouli, M., Voulgari, E., Piperaki, E., Zarrilli, R., et al. (2011). Evolution of Multidrug-Resistant Acinetobacter Baumannii Clonal Lineages: A 10 Year Study in Greece (2000-09). J. Antimicrob. Chemother. 66, 2767-2772. doi: 10.1093/jac/dkr390

Higgins, P., Wisplinghoff, H., Stefanik, D., and Seifert, H. (2004). In Vitro Activities of the $\beta$-Lactamase Inhibitors Clavulanic Acid, Sulbactam, and Tazobactam Alone or in Combination With $\beta$-Lactams Against Epidemiologically Characterized Multidrug-Resistant Acinetobacter Baumannii Strains. Antimicrob. Agents Chemother. 48, 1586-1592. doi: 10.1128/ AAC.48.5.1586-1592.2004

Jeon, J., Lee, J., Lee, J., Park, K., Karim, A., Lee, C., et al. (2015). Structural Basis for Carbapenem-Hydrolyzing Mechanisms of Carbapenemases Conferring Antibiotic Resistance. Int. J. Mol. Sci. 16, 9654-9692. doi: 10.3390/ ijms16059654

Lee, C., Lee, J., Park, M., Park, K., Bae, I., Kim, Y., et al. (2017). Biology of Acinetobacter Baumannii: Pathogenesis, Antibiotic Resistance Mechanisms, and Prospective Treatment Options. Front. Cell. Infect. Microbiol. 7, 55. doi: $10.3389 /$ fcimb.2017.00055

Lemos, E., de la Hoz, F., Einarson, T., McGhan, W., Quevedo, E., Castañeda, C., et al. (2014). Carbapenem Resistance and Mortality in Patients With Acinetobacter Baumannii Infection: Systematic Review and Meta-Analysis. Clin. Microbiol. Infect. 20, 416-423. doi: 10.1111/1469-0691.12363

Lötsch, F., Albiger, B., Monnet, D., Struelens, M., Seifert, H., and Kohlenberg, A. (2020). Epidemiological Situation, Laboratory Capacity and Preparedness for Carbapenem-Resistant Acinetobacter Baumannii in Europe, 2019. Eurosurveillance 25, 2001735. doi: 10.2807/15607917.ES.2020.25.45.2001735

McLeod, S., Moussa, S., Hackel, M., and Miller, A. (2020). In Vitro Activity of Sulbactam-Durlobactam Against Acinetobacter Baumannii-Calcoaceticus
Complex Isolates Collected Globally in 2016 and 2017. Antimicrob. Agents Chemother 64, e02354-19. doi: 10.1128/AAC.02534-19

Nodari, C. S., Santos, F. F., Kurihara, M. N. L., Valiatti, T. B., Cayô, R., and Gales, A. C. (2021). In Vitro Activity of Sulbactam/Durlobactam Against Extensively Drug-Resistant Acinetobacter baumannii Isolates Belonging to South American Major Clones. J. Glob. Antimicrob. Resist. 25, 363-366. doi: 10.1016/j.jgar.2021.05.001

Papp-Wallace, K. M., Senkfor, B., Gatta, J., Chai, W., Taracila, M. A., Shanmugasundaram, V., et al. (2012). Early Insights Into the Interactions of Different $\beta$-Lactam Antibiotics and $\beta$-Lactamase Inhibitors Against Soluble Forms of Acinetobacter Baumannii PBP1a and Acinetobacter Sp. PBP3. Antimicrob. Agents Chemother. 56, 5687-5692. doi: 10.1128/AAC.01027-12

Penwell, W. F., Shapiro, A. B., Giacobbe, R. A., Gu, R. F., Gao, N., Thresher, J., et al. (2015). Molecular Mechanisms of Sulbactam Antibacterial Activity and Resistance Determinants in Acinetobacter baumannii. Antimicrob. Agents Chemother. 59, 1680-1689. doi: 10.1128/AAC.04808-14

Pormohammad, A., Mehdinejadiani, K., Gholizadeh, P., Nasiri, M., Mohtavinejad, N., Dadashi, M., et al. (2020). Global Prevalence of Colistin Resistance in Clinical Isolates of Acinetobacter Baumannii: A Systematic Review and MetaAnalysis. Microb. Pathogenesis 139, p.103887. doi: 10.1016/ j.micpath.2019.103887

Pournaras, S., Dafopoulou, K., Del Franco, M., Zarkotou, O., Dimitroulia, E., Protonotariou, E., et al. (2017). Predominance of International Clone 2 OXA23-Producing-Acinetobacter Baumannii Clinical Isolates in Greece, 2015: Results of a Nationwide Study. Int. J. Antimicrob. Agents 49, 749-753. doi: 10.1016/j.ijantimicag.2017.01.028

Pournaras, S., Gogou, V., Giannouli, M., Dimitroulia, E., Dafopoulou, K., Tsakris, A., et al. (2014). Single-Locus-Sequence-Based Typing of blaOXA-51-Like Genes for Rapid Assignment of Acinetobacter Baumannii Clinical Isolates to International Clonal Lineages. J. Clin. Microbiol. 52, 1653-1657. doi: 10.1128/ JCM.03565-13

Schleicher, X., Higgins, P., Wisplinghoff, H., Körber-Irrgang, B., Kresken, M., and Seifert, H. (2013). Molecular Epidemiology of Acinetobacter Baumannii and Acinetobacter Nosocomialis in Germany Over a 5-Year Period (2005-2009). Clin. Microbiol. Infect 19, 737-742. doi: 10.1111/1469-0691.12026

Seifert, H., Müller, C., Stefanik, D., Higgins, P., Miller, A., and Kresken, M. (2020). In Vitro Activity of Sulbactam/Durlobactam Against Global Isolates of Carbapenem-Resistant Acinetobacter Baumannii. J. Antimicrob. Chemother. 75, 2616-2621. doi: 10.1093/jac/dkaa208

Seifert, H., Stefanik, D., Olesky, M., and Higgins, P. (2020). In Vitro Activity of the Novel Fluorocycline TP-6076 Against Carbapenem-Resistant Acinetobacter Baumannii. Int. J. Antimicrob. Agents 55, 105829. doi: 10.1016/ j.ijantimicag.2019.10.010

Shapiro, A., Moussa, S., McLeod, S., Durand-Réville, T., and Miller, A. (2021). Durlobactam, a New Diazabicyclooctane $\beta$-Lactamase Inhibitor for the Treatment of Acinetobacter Infections in Combination With Sulbactam. Front. Microbiol. 12, 709974. doi: 10.3389/fmicb.2021.709974

Turton, J. F., Gabriel, S. N., Valderrey, C., Kaufmann, M. E., and Pitt, T. L. (2007). Use of Sequence-Based Typing and Multiplex PCR to Identify Clonal Lineages of Outbreak Strains of Acinetobacter Baumannii. Clin. Microbiol. Infect. 13, 807-815. doi: 10.1111/j.1469-0691.2007.01759.x

Viehman, J., Nguyen, M., and Doi, Y. (2014). Treatment Options for CarbapenemResistant and Extensively Drug-Resistant Acinetobacter Baumannii Infections. Drugs 74, pp.1315-1333. doi: 10.1007/s40265-014-0267-8

WHONET (Greece). Available at: http://www.mednet.gr/whonet/.

Wong, D., Nielsen, T., Bonomo, R., Pantapalangkoor, P., Luna, B., and Spellberg, B. (2017). Clinical and Pathophysiological Overview of Acinetobacter Infections: A Century of Challenges. Clin. Microbiol. Rev. 30, 409-447. doi: $10.1128 / \mathrm{cmr} .00058-16$

Xie, R., Zhang, X., Zhao, Q., Peng, B., and Zheng, J. (2018). Analysis of Global Prevalence of Antibiotic Resistance in Acinetobacter Baumannii Infections Disclosed a Faster Increase in OECD Countries. Emerging Microbes Infect 7, 110. doi: $10.1038 / s 41426-018-0038-9$

Yang, Q., Xu, Y., Jia, P., Zhu, Y., Zhang, J., Zhang, G., et al. (2020). In Vitro Activity of Sulbactam/Durlobactam Against Clinical Isolates of Acinetobacter baumannii Collected in China. J. Antimicrob. Chemother. 75, 1833-1839. doi: 10.1093/jac/dkaa119 
Conflict of Interest: This study received funding from Entasis Therapeutics. The funder had the following involvement with the study: provision of MIC plates and next generation sequencing analysis. Entasis was not involved in the collection, analysis, interpretation of susceptibility data, the writing of this article or the decision to submit it for publication. All authors declare no other competing interests.

Publisher's Note: All claims expressed in this article are solely those of the authors and do not necessarily represent those of their affiliated organizations, or those of the publisher, the editors and the reviewers. Any product that may be evaluated in this article, or claim that may be made by its manufacturer, is not guaranteed or endorsed by the publisher.

Copyright (c) 2022 Petropoulou, Siopi, Vourli and Pournaras. This is an open-access article distributed under the terms of the Creative Commons Attribution License (CC BY). The use, distribution or reproduction in other forums is permitted, provided the original author(s) and the copyright owner(s) are credited and that the original publication in this journal is cited, in accordance with accepted academic practice. No use, distribution or reproduction is permitted which does not comply with these terms. 